\title{
Proximidade de rodovias pode influenciar a fragmentação florestal? Um estudo de caso no norte do Rio Grande do Sul
}

\author{
Tiago Rezzadori ${ }^{1}$ \\ Marilia Teresinha Hartmann ${ }^{2}$ \\ Paulo Afonso Hartmann ${ }^{2 *}$ \\ ${ }^{1}$ Universidade Federal da Fronteira Sul, Campus Erechim \\ ${ }^{2}$ Universidade Federal da Fronteira Sul, Campus Erechim \\ Programa de Pós-graduação em Ciência e Tecnologia Ambiental \\ ERS-135 - km 72, 200, Caixa Postal 764, CEP 99700-970, Erechim - RS, Brasil \\ * Autor para correspondência \\ hartmann.paulo@gmail.com
}

Submetido em 19/10/2015

Aceito para publicação em 31/05/2016

\section{Resumo}

O estabelecimento e o uso das rodovias interferem na atmosfera, no solo, na vegetação, na fauna e nas comunidades humanas que estão em seu entorno. Um dos principais efeitos causados pela implantação e operação de uma rodovia é a fragmentação das paisagens naturais, que consequentemente gera o efeito de borda e o isolamento de populações. Nesse sentido, o objetivo deste estudo foi quantificar e comparar a distribuição da cobertura vegetal em áreas com e sem influência ecológica de rodovias na região do Alto Uruguai, norte do Rio Grande do Sul. Para tal, foi criado um banco de dados georrelacional, onde foram inseridas imagens de satélite Landsat V e CBERS 2b. Foi estimado o tamanho e quantidade de fragmentos florestais nas áreas com e sem influência das rodovias. A área com influência ecológica de rodovias apresentou menos fragmentos florestais e de menor tamanho quando comparada com a área sem influência ecológica. A alta fragmentação florestal parece ser potencializada pela proximidade das rodovias na região norte do Rio Grande do Sul, contribuindo para o isolamento e redução do tamanho das populações nativas que ocupam essas áreas.

Palavras-chave: Ecologia de Estradas; Fauna; Mapeamento; Paisagem

\section{Abstract}

Can proximity to roads influence forest fragmentation? A case study in northern Rio Grande do Sul, Brazil. The establishment and use of roads interfere with the atmosphere, soil, vegetation, fauna, and human communities surrounding them. One of the main effects caused by the implementation and operation of a road is fragmentation of natural landscapes, which consequently generates edge effects and isolation of populations. Thus, this study aimed to quantify and compare the distribution of vegetation cover in areas with and without ecological influence of roads in the Alto Uruguai region, northern Rio Grande do Sul, Brazil. To do this, a georelational database was created, where satellite images taken by Landsat V and CBERS $2 \mathrm{~b}$ were entered. The size and amount of forest fragments were estimated in areas with and without influence of roads. The area with 
ecological influence of roads had less and smaller sized forest fragments when compared to the area without ecological influence. High forest fragmentation seems to be enhanced by proximity to roads in northern Rio Grande do Sul, contributing to isolate and reduce the size of native populations that occupy these areas.

Key words: Fauna; Landscape; Mapping; Road Ecology

\section{Introdução}

Entender os impactos ambientais e definir estratégias de conservação da biodiversidade estão entre os maiores desafios a serem enfrentados pela comunidade científica na atualidade, em função do elevado nível de perturbações antrópicas, em diferentes escalas, nos ecossistemas naturais (VIANA; PINHEIRO, 1998). Neste sentido, estudar a dinâmica dos fragmentos florestais ganha importância na medida em que grande parte da biodiversidade se encontra em pequenos remanescentes florestais, resultantes da fragmentação de áreas maiores (FORMAN; GODRON, 1986; GASCON et al., 2001).

A fragmentação de uma floresta é caracterizada pelo processo no qual uma área contínua é tanto reduzida em sua superfície quanto dividida em dois ou mais fragmentos. A redução da área de hábitat disponível pode levar a uma redução na diversidade biótica local (TURNER, 1989; METZGER, 1999). Isso pode ocorrer diretamente, pela perda da área, ou em longo prazo, por meio dos efeitos de singularização. Uma consequência inevitável da fragmentação florestal é um drástico aumento no total de bordas de hábitat. Consequentemente, as populações animais e vegetais presentes nos fragmentos não estão apenas reduzidas e subdivididas, mas também são expostas a mudanças abióticas e bióticas associadas à borda das florestas (LAURANCE et al., 1997).

As estradas estão ligadas diretamente a impactos ambientais, pois podem induzir mudanças na cobertura florestal (LAURANCE et al., 2009). Para Forman et al. (2003), as rodovias são agentes de fragmentação de alto impacto que afetam ambientes físicos, químicos e biológicos de um ecossistema. As principais peculiaridades de uma rodovia como agente de fragmentação estão relacionadas com a própria superfície e com o tráfego de veículos (PIRES et al.,
2002). Os efeitos negativos causados pelas rodovias são evidenciados nas comunidades vegetais com a fragmentação das áreas (NAGENDRA et al., 2003; LAURANCE et al., 2009). As rodovias tornam-se mecanismos de alto impacto por remover a cobertura vegetal original e alterar a função e a estrutura da paisagem (FORMAN; DEBLINGER, 2000). Ao gerar alterações nas relações ecológicas, os impactos mais evidentes são a perda de hábitats, fragmentação e mortalidade de fauna por atropelamento (FORMAN; ALEXANDER, 1998; CLEVENGER et al., 2003; COELHO et al., 2008; ROSA; BAGER, 2013). A fragmentação, em função dos efeitos de borda e barreira, pode acarretar a inacessibilidade a recursos e divisão das populações (LAURANCE et al., 1997; 2008). No entanto, a magnitude das consequências causadas por esses efeitos vai depender das exigências ecológicas de cada espécie e da forma que o fragmento possui (CASTRO, 2008).

A proximidade de rodovias de fragmentos florestais pode causar aumento dos efeitos de borda e, consequentemente, mudar a composição das comunidades (FREITAS et al., 2014). Sob essa perspectiva, em estudos de paisagens fragmentadas, devem-se considerar as rodovias quanto à sua distância em relação ao fragmento de hábitat ou quanto à sua densidade na paisagem ou no entorno do fragmento (FREITAS; METZGER, 2007). Informações sobre presença e a distância das rodovias podem ser de grande utilidade na delimitação de áreas prioritárias para conservação e restauração ambiental (WILLIAMS et al., 2002; CABEZA, 2003; SOUSA et al., 2009).

A região norte do estado do Rio Grande do Sul tem na agropecuária e na agroindústria sua base produtiva (COTRIM, 2014). A grande maioria dos municípios da região caracteriza-se pela produção em pequenos e médios estabelecimentos rurais, apoiados no sistema de agricultura familiar (PIRAN, 2001). O cenário de 
intensa exploração dos recursos naturais resulta em fragmentação da paisagem, que é acentuada pelo uso das rodovias e pela necessidade de escoar a produção. Neste sentido, o objetivo deste estudo foi quantificar e comparar a distribuição da cobertura vegetal em áreas com e sem influência ecológica de rodovias na região do Alto Uruguai, no norte do estado do Rio Grande do Sul. Estas informações podem colaborar para proposição de medidas que visem à redução dos impactos negativos da fragmentação na região.

\section{Material e Métodos}

\section{Área do estudo}

A região norte do estado do Rio Grande do Sul foi marcada historicamente pelo desmatamento intenso e consequente fragmentação ambiental (MARTINAZZO, 2011). Sua rede viária perpassa relevos planos e ondulados, rios, fragmentos florestais e áreas protegidas. A vegetação local apresenta características de dois tipos de formações florestais: Floresta Ombrófila Mista e
Floresta Estacional Decidual. A vegetação é composta por uma diversidade de espécies distribuídas ao longo dos remanescentes. As altitudes variam de $100 \mathrm{~m}$ (nas calhas dos rios) a $800 \mathrm{~m}$ nos topos dos morros. O terreno é ondulado e os vales bem marcados ao longo dos rios principais e seus afluentes (RIO GRANDE DO SUL, 2010). A região integra parte da área da Reserva da Biosfera da Mata Atlântica e inclui, dentro de seus limites, Unidades de Conservação, Áreas Indígenas, Sítios Arqueológicos e Comunidades Quilombolas.

A área de amostragem foi delimitada utilizando-se unidades de paisagem definidas pela Secretaria Estadual do Meio Ambiente (SEMA). Foram escolhidas partes das unidades de paisagem PM1 (Planalto Meridional), que abrange as Bacias Hidrográficas de Passo Fundo, Turvo - Santa Rosa - Santo Cristo, Várzea, e da unidade de paisagem PM3, que é composta pelas bacias hidrográficas de Passo Fundo e Apuaê-Inhandava (RIO GRANDE DO SUL, 2010). As Unidades de Paisagem escolhidas para o estudo compreendem a parte norte do Rio Grande do Sul, denominada Alto Uruguai gaúcho (Figura 1).

FIGURA 1: Localização da área de estudo, região norte do estado do Rio Grande do Sul.

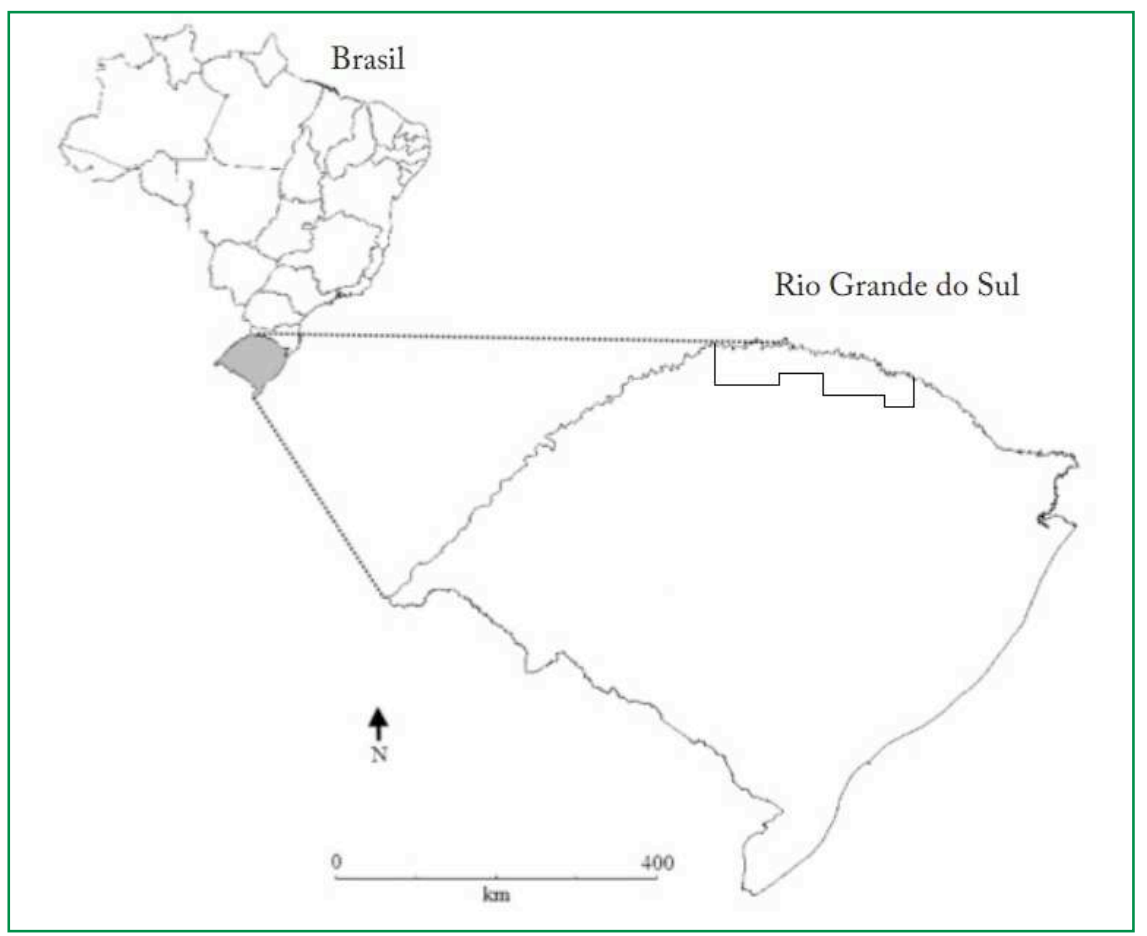

Fonte: Autores. 


\section{Coleta de dados}

Foi montado um banco de dados georrelacional em um Sistema de Informações Geográficas (SIG), cuja montagem foi feita com o auxílio do software GPS Trackmaker. Nesse banco georrelacional, foram inseridas as imagens orbitais do satélite Landsat V e CBERS 2b (sensor/câmara HRC - com resolução espacial de 2,4 m) para realizar a extração das informações sobre a disposição das rodovias. Foram demarcadas todas as rodovias pavimentadas na região do estudo. Para estimar a área de influência ecológica das rodovias, foi seguida a metodologia indicada em Forman e Deblinger (2000), que considera o tamanho da faixa de influência de $200 \mathrm{~m}$ para rodovias pavimentadas simples, que predominam na região. Após estabelecer a área com influência ecológica das rodovias (0 a $200 \mathrm{~m}$ da rodovia), foi delimitada outra área, considerada sem influência ecológica da rodovia, com distância de aproximadamente 800 a $1000 \mathrm{~m}$ da rodovia. Dentro das áreas foram demarcados os fragmentos florestais com tamanho maior ou igual a 0,4 ha e contado o número e medido o tamanho destes.

\section{Análises}

Com a definição da área total amostrada, foi estimada a densidade de rodovias para a região (comprimento total das rodovias/área total). Foi estimado o tamanho das áreas com e sem influência ecológica das rodovias e calculada a percentagem da área total amostrada que está sob influência ecológica das rodovias. Foi estimada a porcentagem de cobertura florestal em cada área e calculado o tamanho médio dos fragmentos florestais. Como os dados não seguem um padrão paramétrico, utilizou-se o teste estatístico Mann-Whitney com o auxílio do Software Statistica 7 para verificar se existe diferença nos tamanhos médios dos fragmentos nas áreas com e sem influência ecológica.

\section{Resultados}

A área total avaliada corresponde a 1.016.000 ha $\left(10.160 \mathrm{~km}^{2}\right)$. Nessa área, foram registrados 964,73 $\mathrm{km}$ de rodovias simples pavimentadas. A densidade de rodovias simples pavimentadas na área amostrada foi de $0,0950 \mathrm{~km}$ de rodovia por $\mathrm{km}^{2}$. A área de influência ecológica total é de 44.209 ha $\left(442,09 \mathrm{~km}^{2}\right)$, ou seja, $4,35 \%$ da área total amostrada. A cobertura florestal na área com influência ecológica da rodovia é de 8.811 ha $\left(88,11 \mathrm{~km}^{2}\right)$, o que representa uma $19,93 \%$. Na área sem influência ecológica das rodovias, a cobertura florestal é de 9.981 ha $\left(99,81 \mathrm{~km}^{2}\right)$, e representa uma cobertura florestal de 23,09\%.

$\mathrm{Na}$ área com influência ecológica da rodovia, foram identificados 670 fragmentos florestais (Tabela 1; Figura 2), e estes possuem um tamanho médio de 13,15 ha. Na área sem influência da rodovia, foram encontrados 703 fragmentos florestais (Tabela 1; Figura 3), com um tamanho médio de 14,20 ha. O tamanho médio dos fragmentos florestais é significativamente maior na área sem influência das rodovias (Mann-Whitney $\mathrm{U}$ test statistic $=212885,0 ; p=0,0021 ;$ Figura 4).

A área sem influência ecológica das rodovias tem mais fragmentos e concentrados em tamanhos maiores quando comparada à área com influência ecológica das rodovias. Nas duas áreas, a maior parte dos fragmentos foi encontrada no corte de menor tamanho, entre $0,4-$ 9,99 ha (Tabela 1). No entanto, a área sem influência ecológica das rodovias possui 10\% menos fragmentos com área de 0 a 9,99 ha que na área com influência ecológica das rodovias. Para os cortes de 10 - 19,99; 20 -29,99 e 30 - 39,99 ha, a área sem influência ecológica das rodovias possui maior quantidade de fragmentos (Tabela 1). 
TABELA 1: Distribuição do tamanho dos fragmentos florestais nas áreas com e sem influência ecológica das rodovias na região norte do estado do Rio Grande do Sul.

\begin{tabular}{ccc}
\hline $\begin{array}{c}\text { Tamanho dos fragmentos } \\
\text { (ha) }\end{array}$ & $\begin{array}{c}\text { Área com influência } \\
\text { n (\%) }\end{array}$ & $\begin{array}{c}\text { Área sem influência } \\
\mathbf{n}(\%)\end{array}$ \\
\hline $0,4-9,99$ & $392(58,51)$ & $343(48,79)$ \\
$10-19,99$ & $155(23,13)$ & $192(27,31)$ \\
$20-29,99$ & $67(10,00)$ & $92(13,09)$ \\
$30-39,99$ & $23(3,43)$ & $44(6,26)$ \\
$40-49,99$ & $15(2,24)$ & $14(1,99)$ \\
$50-59,99$ & $9(1,34)$ & $11(1,56)$ \\
$60-69,99$ & $3(0,45)$ & $4(0,57)$ \\
$70-79,99$ & $1(0,15)$ & $1(0,14)$ \\
$80-89,99$ & $1(0,15)$ & $1(0,14)$ \\
$90-99,99$ & $0(0,00)$ & $0(0,00)$ \\
$100-199,99$ & $3(0,45)$ & $0(0,00)$ \\
$>200$ & $1(0,15)$ & $1(0,14)$ \\
\hline Total & $\mathbf{6 7 0 ( 1 0 0 )}$ & $\mathbf{7 0 3}(\mathbf{1 0 0})$ \\
\hline
\end{tabular}

FIGURA 2: Distribuição dos tamanhos dos fragmentos florestais na área com influência ecológica das rodovias na região norte do estado do Rio Grande do Sul. Fragmentos com área maior que 200 ha não são apresentados.

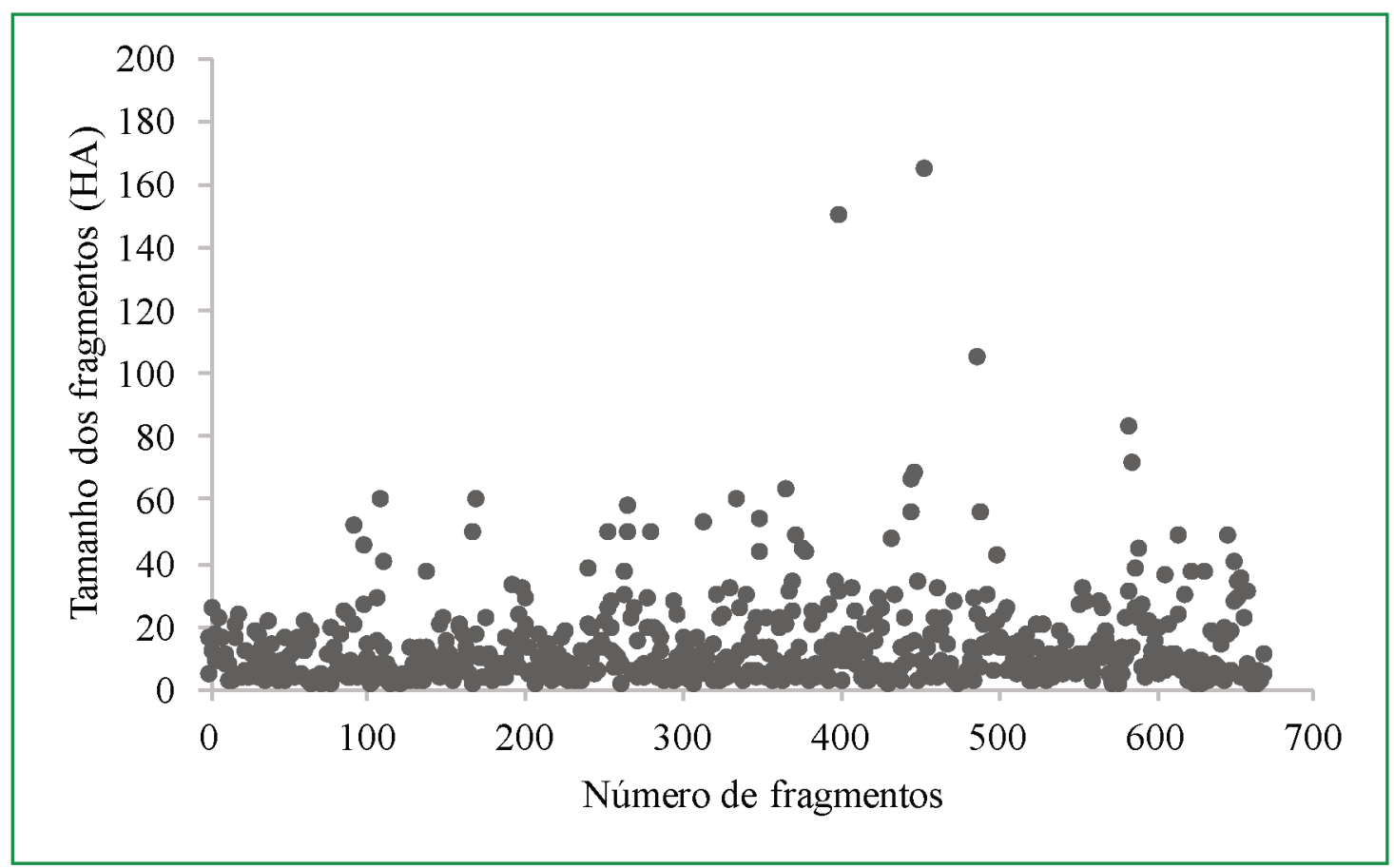


FIGURA 3: Distribuição dos tamanhos dos fragmentos florestais na área sem influência ecológica das rodovias na região norte do estado do Rio Grande do Sul. Fragmentos com área maior que 200 ha não são apresentados.

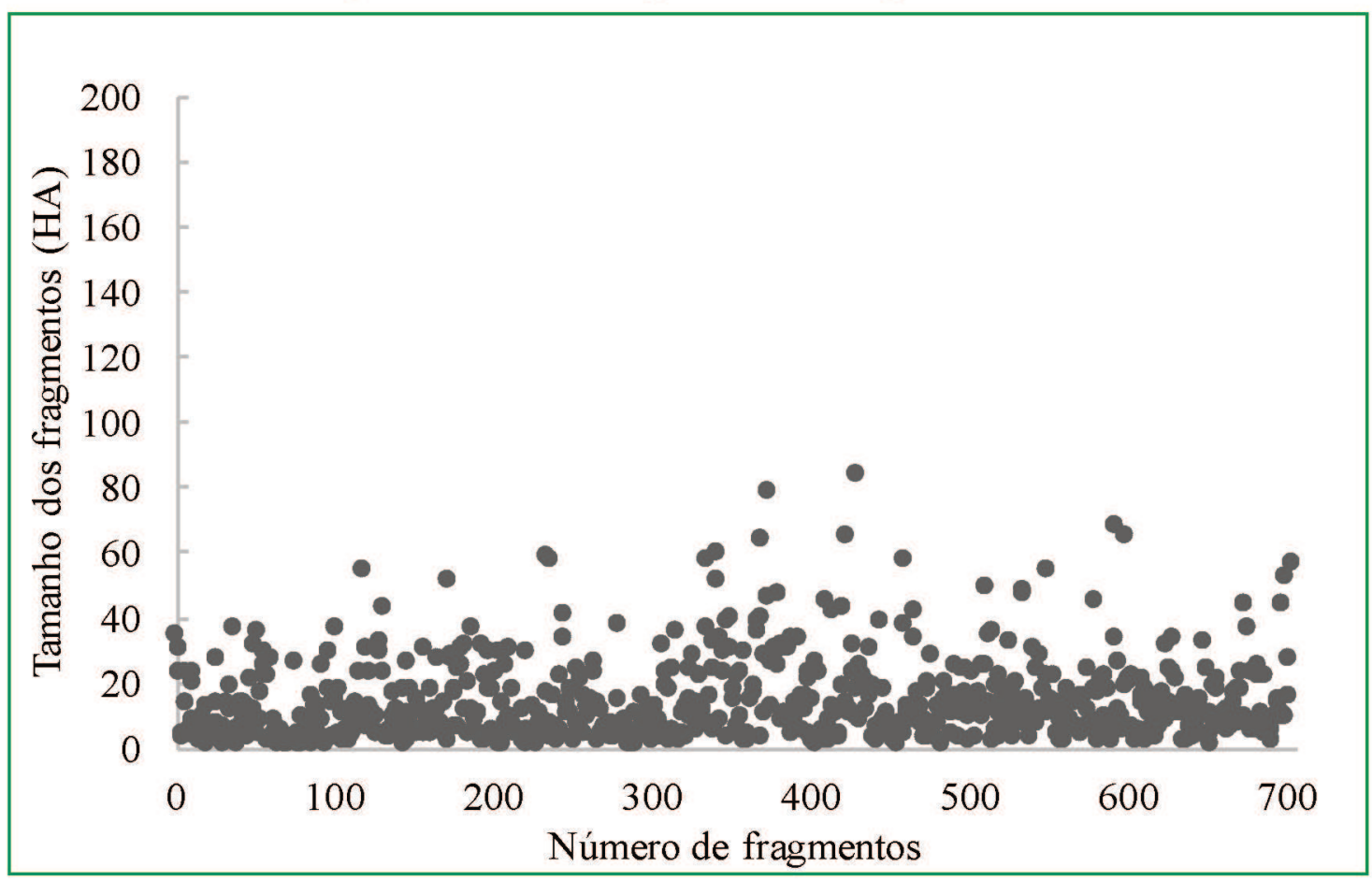

FIGURA 4: Tamanho dos fragmentos florestais nas áreas com (1) e sem (2) influência ecológica das rodovias, na região norte do estado do Rio Grande do Sul.

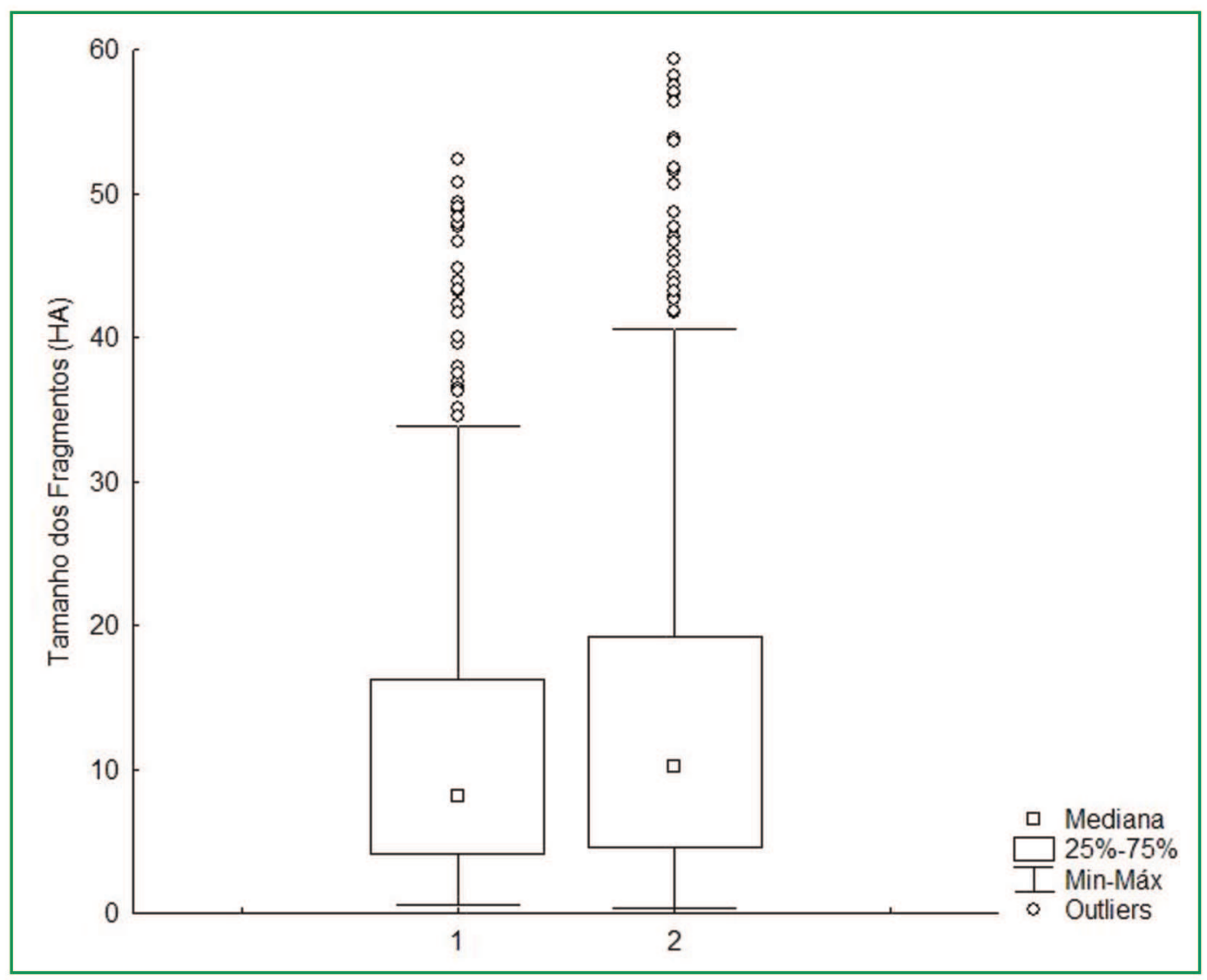




\section{Discussão}

A instalação de rodovias modifica e altera a estrutura das paisagens, constituindo atualmente um dos principais agentes de fragmentação, seja de forma direta, pela construção e operação de rodovias, ou indireta, por facilitar ocupação de novas áreas pelos humanos (FORMAN; DEBLINGER, 2000). Neste sentido, a menor cobertura florestal na área com influência ecológica das rodovias pode estar associada a diferentes fatores. O primeiro é o objetivo principal das rodovias, que é manter a ligação entres cidades e localidades e servir de apoio para escoar a produção. Desta forma, áreas próximas às rodovias tendem a ter maior uso do solo destinado a atividades como agricultura, reflorestamento ou urbanização (FORMAN et al., 2003; FREITAS et al., 2010). Além disto, o relevo mais plano, onde as rodovias são normalmente implantadas, favorece a realização de atividades antrópicas (POLITANO, 1989), que consequentemente reduzem a cobertura florestal e aumentam a fragmentação florestal. Neste sentido, fatores como declividade e tipo de uso da terra podem influenciar de forma integrada as rodovias no processo de fragmentação na região, assim como estradas rurais e de pequeno porte.

Outro ponto relevante é o elevado grau de fragmentação florestal nas áreas amostradas, principalmente nas mais próximas às rodovias. $\mathrm{O}$ menor número de fragmentos florestais e com áreas menores em locais mais próximos das rodovias contribuem para a hipótese de que quanto mais perto da rodovia, maior a fragmentação florestal. $\mathrm{O}$ processo de fragmentação leva à formação de uma paisagem em mosaico e age fundamentalmente reduzindo e isolando as áreas propícias à sobrevivência das populações (METZGER, 1999). Os efeitos negativos ocorrem, principalmente, em razão da perda e fragmentação de hábitats (FORMAN; DEBLINGER 2000), exercendo influência sobre a dinâmica da fauna local, interferindo na distribuição e estrutura das populações (BISSONETTE; ROSA, 2009; BAGER; FONTANA, 2012).

O tamanho bem como a distância entre os fragmentos são fatores críticos na manutenção das populações e na determinação da riqueza de espécies.
A redução no tamanho de um fragmento implica em reduções no tamanho das populações, e pequenos tamanhos populacionais aumentam a probabilidade de extinção (SAUNDERS et al., 1991). O impacto do efeito de borda pode variar em função das características da paisagem de cada região ou em função do agente promotor da borda (e.g. rodovias, pecuária e agricultura). Em bordas geradas pela presença de uma rodovia, as comunidades biológicas tendem a ser modificadas e simplificadas em termos de número de espécies e indivíduos (FORMAN et al., 2003; LAURANCE et al., 2009). Na área de estudo, populações que ocupam áreas mais próximas das rodovias estão mais sujeitas a processos de redução populacional e possível extinção local, em consequência da redução e fragmentação de áreas florestadas.

Esses dados reforçam a necessidade de cautela na implantação e operação de rodovias que perpassem áreas protegidas. A redução na cobertura florestal e no tamanho dos fragmentos associada à alta fragmentação provocada a partir da presença e operação de rodovias têm consequências negativas na persistência das populações no entorno da rodovia. Na área do estudo nenhuma Unidade de Conservação é afetada diretamente pelas rodovias avaliadas. No entanto, o maior fragmento florestal contíguo pertence à área protegida Terra Indígena Nonoai Rio da Várzea, nos municípios de Planalto, Gramado dos Loureiros e Nonoai. Essa área é cortada pela ERS-324, o que intensifica o efeito de borda (JACKSON; FAHRIG, 2011) e reduz a proporção de área efetivamente preservada. Nossos dados indicam que a alta fragmentação florestal da região parece ser potencializada pela proximidade das rodovias na região norte do estado do Rio Grande do Sul, contribuindo para o isolamento e redução no tamanho das populações nativas.

\section{Agradecimentos}

Agradecemos a Jéssica Mulinari, Cassiane Kolcenti, Siane Camila Luzzi e Fabio de Oliveira Sanches pelo auxílio durante a coleta e análise de dados. A UFFS pelo apoio na forma de bolsa a Tiago Rezzadori (Edital 218/UFFS/2013). 


\section{Referências}

BAGER, A.; FONTOURA, V. Ecologia de estradas no Brasil: contexto histórico e perspectivas futuras. In: BAGER A. (Org.). Ecologia de estradas: tendências e pesquisas. Lavras: UFLA, 2012. p. 13-33.

BISSONETTE, J. A.; ROSA S. A. Road zone effects in smallmammal communities. Ecology and Society, Wolfville, v. 14 (1), n. 27, 2009. [online] URL: <http://www.ecologyandsociety.org/vol14/ iss $1 / \mathrm{rt} 27 />$.

CABEZA, M. Habitat loss and connectivity of reserve networks in probability approaches to reserve design. Ecology Letters, Montpellier, v. 6, p. 665-672, 2003.

CASTRO, D. Efeitos de borda em ecossistemas tropicais: síntese bibliográfica e estudo de caso em fragmento de cerrado, na região nordeste do estado de São Paulo. 2008. Dissertação (Mestrado em Ciências) - Instituto de Biociência da Universidade de São Paulo, São Paulo. 2008.

CLEVENGER, A. P.; CHRUSZCZ, B.; GUNSON, K. E. Spatial patterns and factors influencing small vertebrate fauna road-kill aggregations. Biological Conservation, Boston, v. 109, p. 15-26, 2003.

COELHO, I. P.; KINDEL, A.; COELHO, A. V. P. Roadkills of vertebrate species on two highways through the Atlantic Forest Biosphere Reserve, southern Brazil. European Journal of Wildlife Research, Berlin, v. 54, p. 689-699, 2008.

COTRIM, D. Desenvolvimento rural e agricultura familiar: produção acadêmica da Ascar. Porto Alegre: Emater/Ascar, 2014. Disponível em: <http://www.emater.tche.br/site/arquivos_pdf/ teses//E_Book3.pdf $>$. Acesso em: 10 out. 2015.

FORMAN, R. T.; ALEXANDER, L. E. Roads and their major ecological effects. Annual Review of Ecology, Evolution and Systematics, Palo Alto, v. 29, p. 207-231, 1998.

FORMAN, R. T. T.; DEBLINGER, R. D. The ecological road-effect zone of a Massachusetts (USA) suburban highway. Conservation Biology, San Francisco, v. 14, p. 36-46, 2000.

FORMAN, R. T. T.; GODRON, R. Landscape Ecology. New York: John Wiley \& Sons, 1986. 712 p.

FORMAN, R. T. T.; SPERLING, D.; BISSONETTE, J. A.; CLEVENGER, A. P.; CUTSHALL, C. D.; DALE, V. H.; FAHRIG, L.; FRANCE, R.; GOLDMAN, C. R.; HEANUE, K.; JONES, J. A.; SWANSON, F. J.; TURRENTINE, T.; WINTER. T. C. Road ecology: science and solutions. Washington: Island Press, 2003. $481 \mathrm{p}$.

FREITAS, S. R.; HAWBAKER, T. J.; METZGER, J. P. Effects of roads, topography, and land use on forest cover dynamics in the Brazilian Atlantic Forest. Forest Ecology and Management, Amsterdam, v. 259, p. 410-417, 2010.

FREITAS, S. R; METZGER, J. P. Relação entre a densidade e a conectividade das estradas, e o relevo em uma paisagem fragmentada da Mata Atlântica (Planalto de Ibiúna, SP). In: SIMPÓSIO BRASILEIRO DE SENSORIAMENTO REMOTO, XIII, 2007, Florianópolis. Resumos... Florianópolis: INPE, 2007. p. 2659-2661. FREITAS, S. R.; SOUSA, C. O. M.; BOSCOLO, D.; METZGER J. $P$. How are native vegetation and reserves affected by different road types in a southeastern Brazilian state? Oecologia Australis, Rio de Janeiro, v. 17, n. 44, p. 447-458, 2014.

GASCON, C.; LAURENCE, W. F.; LOVEJOY, T. Fragmentação florestal e biodiversidade na Amazônia Central. In: GARAY, I.;
DIAS, B. (Org.). Conservação da biodiversidade em ecossistemas tropicais. Petrópolis: Vozes, 2001. p. 29-42.

JACKSON, N. D.; FAHRIG. L. Relative effects of road mortality and decreased connectivity on population genetic diversity. Biological Conservation, Boston, v. 144, n. 12, p. 3143-3148, 2011.

LAURANCE, W. F.; CROES, B. M.; GUISSOUEGOU, N.; BUIJ, R.; DETHIER, M.; ALONSO, A. Impacts of roads, hunting, and habitat alteration on nocturnal mammals in African rainforest. Conservation Biology, San Francisco, v. 22, p. 721-732, 2008.

LAURANCE, W. F.; GASCON, C.; RANKIN-DE MERON, J. M. Predicting effects of habitat destruction on plant communities: a test of a model using Amazonian trees. Ecological Applications, Ithaca, v. 9, n. 2, p. 548-554, 1997.

LAURANCE, W. F.; GOOSEM, M.; LAURANCE, S. G. Impacts of roads and linear clearings on tropical forests. Trends in Ecology and Evolution, Cambridge, v. 24, n. 12, p. 659-699, 2009.

MARTINAZZO, L. N. História ambiental do Alto Uruguai: colonização, desenvolvimento e transformações na paisagem. 2011. 1001 f. Dissertação (Mestrado em Ambiente e Desenvolvimento) Centro Universitário UNIVATES, Lajeado. 2011.

METZGER, J. P. Estrutura da paisagem e fragmentação: análise bibliográfica. Anais da Academia Brasileira de Ciências, Rio de Janeiro, v. 71, n. 3, p. 445-463, 1999.

NAGENDRA, H.; SOUTHWORTH, J.; TUCKER, C. Accessibility as a determinant of landscape transformation in western Honduras: linking pattern and process. Landscape Ecology, Oxford, v. 18, p. 141-158, 2003.

PIRAN, N. Agricultura familiar: lutas e perspectivas no Alto Uruguai. Erechim: EdiFAPES, 2001. 190 p.

PIRES, A. S.; LIRA, P. K.; FERNANDEZ, F. A. S.; SCHITTINI, G. M.; OLIVEIRA, L. C. Frequency of movements of small mammals among Atlantic Coastal Forest fragments in Brazil. Biological Conservation, Boston, v. 108, p. 229-237, 2002.

POLITANO, W. O papel das estradas na economia rural. São Paulo: Nobel, 1989. 78 p.

RIO GRANDE DO SUL. Zoneamento ambiental da silvicultura: diretrizes da silvicultura por unidade de paisagem e bacia hidrográfica. Porto Alegre: Secretaria Estadual do Meio Ambiente, 2010. 300 p.

ROSA, C. A.; BAGER, A. Review of the factors underlying the mechanisms and effects of roads on vertebrates. Oecologia Australis, Rio de Janeiro, v. 17, n. 1, p. 6-19, 2013.

SAUNDERS, D. A.; HOBBS, R. J.; MARGULES C. R. Biological consequences of ecosystem fragmentation: a review. Conservation Biology, San Francisco, v. 5, n. 1, p. 18-32, 1991.

SOUSA, C. O. M.; FREITAS, S. R.; DIAS, A. A.; GODOY, A. B. P.; METZGER, J. P. O papel das estradas na conservação da vegetação nativa no estado de São Paulo. In: SIMPÓSIO BRASILEIRO DE SENSORIAMENTO REMOTO, XVI, 2009, Natal. Resumos... Natal: INPE, 2009. p. 3087-3094.

TURNER, M. G. Landscape ecology: the effect of pattern on process. Annual Reviews in Ecology \& Systematics, Palo Alto, v. 20, p. 171-197, 1989.

VIANA, V. M.; PINHEIRO, L. A. F. V. Conservação da biodiversidade em fragmentos florestais. Instituto de Pesquisas e Estudos Florestais, Piracicaba, v. 12, n. 32, p. 25-42, 1998.

WILliamS, P. H.; MARGULES, C. R.; HILBERT, D. W. Data requirements and data sources for biodiversity priority area selection. Journal of Biosciences, Karnataka, v. 27, n. 4, p. 327-338, 2002. 